\title{
Microglia and C9orf72 in neuroinflammation and ALS and frontotemporal dementia
}

\author{
Deepti Lall' and Robert H. Baloh ${ }^{1,2}$ \\ 'Board of Governors Regenerative Medicine Institute and 2Department of Neurology, Cedars-Sinai Medical Center, Los Angeles, California, USA.
}

\begin{abstract}
Amyotrophic lateral sclerosis (ALS) is a degenerative disorder that is characterized by loss of motor neurons and shows clinical, pathological, and genetic overlap with frontotemporal dementia (FTD). Activated microglia are a universal feature of ALS/FTD pathology; however, their role in disease pathogenesis remains incompletely understood. The recent discovery that ORF 72 on chromosome 9 (C9orf72), the gene most commonly mutated in ALS/FTD, has an important role in myeloid cells opened the possibility that altered microglial function plays an active role in disease. This Review highlights the contribution of microglia to ALS/FTD pathogenesis, discusses the connection between autoimmunity and ALS/FTD, and explores the possibility that C9orf72 and other ALS/FTD genes may have a "dual effect" on both neuronal and myeloid cell function that could explain a shared propensity for altered systemic immunity and neurodegeneration.
\end{abstract}

\section{Introduction}

Amyotrophic lateral sclerosis (ALS), also known as Lou Gehrig's disease, is a progressive neurodegenerative disorder characterized by loss of motor neurons in motor cortex, brainstem, and spinal cord that results in muscle weakness and atrophy, spasticity, and compromised speech, swallowing, and breathing. ALS is an invariably fatal disease, with patients typically dying within 3 to 5 years after symptom onset $(1,2)$. Most ALS cases are sporadic; i.e., patients do not have a family history of ALS and the cause of their disease is unknown. However, about $5 \%-10 \%$ of ALS cases have a family history of the disorder, typically with dominant inheritance. During the past decades, pathogenic mutations in a number of genes, including ORF 72 on chromosome 9 (C9orf72), superoxide dismutase 1 (SOD1), TAR DNA binding protein (TARDBP, also known as TDP-43), FUS RNA binding protein (FUS), heterogeneous nuclear ribonucleoprotein A1 (HNRNPA1), sequestosome 1 (SQSTM1), valosin-containing protein (VCP), optineurin (OPTN), TANK binding kinase 1 (TBK1), ubiquilin 2 (UBQLN2), and profilin 1 (PFN1), have been identified in ALS (3-18). These genes are involved in a variety of cellular pathways, including protein homeostasis, RNA metabolism, and cytoskeletal dynamics. Importantly, many apparently sporadic cases of ALS also have a genetic component: $1 \%-3 \%$ of sporadic ALS patients carry missense mutations in SOD1 (19), and roughly 5\%-10\% of the sporadic ALS are caused by intronic expansions in C9orf72 (20), with a larger number of cases carrying missense variants of unclear significance in one or more ALS genes (21). Several additional genetic variants have also been discovered that increase susceptibility to ALS and/or modify the clinical phenotype. For example, intermediate expansions to 27-33 CAG repeats in ataxin 2 (ATXN2) increase the risk of developing ALS (22), whereas reduced expression of the axonal guidance gene EPH receptor A4 (EPHA4) is

Conflict of interest: The authors have declared that no conflict of interest exists. Reference information: / Clin Invest. 2017;127(9):3250-3258.

https://doi.org/10.1172/JCI90607. associated with improved overall survival of people with ALS (23). These genetic insights provide the conceptual basis for most ideas regarding mechanisms underlying the initiation and progression of this devastating disease.

Using cellular and animal model systems based on genetic insights, a picture of the etiology and pathogenesis of ALS has started to develop, although the priming events that lead to disease initiation and mechanisms causing motor neuron degeneration still remain elusive. Numerous studies support the idea that neuronal cell death is multifactorial and includes mechanisms such as endoplasmic reticulum stress, mitochondrial dysfunction, oxidative stress, misfolded protein aggregation and impaired degradation, calcium overload, aberrant RNA/DNA regulation, and neuroinflammation $(24,25)$. Neuroinflammation is a pathological hallmark of ALS patients, and PET studies have shown that activated microglia are present in the brains of living ALS patients (26, 27). Early studies on ALS patient autopsies revealed that activated astrocytes, microglia, and infiltrating $\mathrm{T}$ cells are found at sites of motor neuron injury (28-30), suggesting that the immune system might have a role to play in the disease. More recently, identification of several ALS/frontotemporal dementia (FTD) genes, such as C9orf72, progranulin (PGRN), and TBK1, which are highly expressed in microglia and influence their function, has highlighted a potential direct role for these cells in ALS/FTD pathogenesis $(18,31-33)$. In this Review, we focus on the role of immune cells in the pathogenesis of ALS/FTD, and discuss epidemiological evidence for overlap between autoimmunity and ALS/FTD, suggesting shared genetic risk factors in these diseases.

\section{Genetic connections between microglia and neurodegeneration}

Microglia are the main cells in the CNS responsible for immune surveillance and are critical for normal development and homeostasis (34-36). Under steady-state conditions, "ramified" microglia continuously survey the local microenvironment for any foreign antigens and insults. The resident microglia are 
derived from early yolk sac precursors (37), and are distinct from circulating monocytes that can infiltrate into the brain in the setting of injury or neurodegeneration (38). Any insult or injury that disrupts brain homeostasis is sensed rapidly by microglia, resulting in a change in their morphology, and release of cytokines and chemokines to clear pathogens or debris in order to mitigate the damage caused by the injury (35). Upon insult, microglia upregulate expression levels of certain molecules, such as CD11b and Iba1, and gain expression of molecules associated with antigen presentation, such as MHC-II, B7.1 (CD80), and B7.2 (CD86), which are absent in naive microglia (39). However, under chronic stress conditions such as progressive neurodegeneration and breakdown of the blood-brain barrier, there is an infiltration of other myeloid cells into the brain (39). Elegant studies have shown that these infiltrating cells present different gene expression profiles and are phenotypically different from resident microglia (39-41), but work together with the resident microglia to control the damage inflicted due to danger stimuli. Microglia with a morphologically activated phenotype are present in nearly all CNS diseases, including Alzheimer's disease (AD), Parkinson's disease, ALS/FTD, and prion diseases $(42,43)$. Given the nonspecific nature of microglial activation in neurodegeneration, it has long been debated whether it is a cause or a consequence of disease. However, the idea that microglia may play an active role in neurodegeneration has been strongly reinforced in recent years by the discovery of genetic variants in several immune genes that alter the risk of neurodegenerative diseases (44-46). Heterozygous mutations in triggering receptor expressed on myeloid cells 2 (TREM2) are known to increase the risk for various neurodegenerative disorders, including $\mathrm{AD}$ and possibly also ALS (47-49). TREM2 is exclusively expressed by microglia in the CNS $(48,50)$, and mutations in this gene likely confer loss of TREM2 protein function, thereby leading to altered microglial survival, phagocytosis, and inflammatory response $(51,52)$. Similarly, haploinsufficiency in another microglial-expressed gene, $P G R N$, is a common genetic cause of FTD, and also confers elevated risk of developing AD (53). PGRN acts as an inflammatory modulator and facilitates microglial phagocytosis of amyloid- $\beta$ $(\mathrm{A} \beta)$ and apoptotic cells $(54,55)$. Lack of PGRN leads to dysregulation of microglial complement gene expression and loss of synaptic pruning and lysosome maturation (56). Several other genetic risk factors have also been identified that affect immune regulation and development of neurodegeneration. Leucine-rich repeat kinase 2 (LRRK2), which is mutated in Parkinson's disease, is highly expressed in activated microglia and peripheral myeloid cells and is associated with several immune-related disorders (57). Apolipoprotein E allele $\varepsilon 4\left(A P E^{*} \varepsilon 4\right)$, the strongest known genetic risk factor for $\mathrm{AD}$, is highly expressed in microglia and has been shown to influence microglial activation in response to A $\beta$ deposition (58). Microglia also express complement receptors and mediate synaptic pruning in the developing brain $(59,60)$. Classical complement protein $\mathrm{C} 1 \mathrm{q}$ is induced in the brains of $\mathrm{AD}$ mice $(61,62)$ and mediates early synapse loss in AD mouse models (63). Interestingly, secretion of inflammatory proteins such as $\mathrm{C} 1 \mathrm{q}$ and IL- $1 \alpha$ by activated microglia leads to activation of astrocytes, which may be neurotoxic in neurodegenerative disorders (64). Polymorphisms in the genes encoding complement receptor 1 (CR1) (65) and clusterin (66) have also been hypothesized to influence receptor-mediated clearance of $A \beta$ from the brain by microglial endocytosis. Subsequent studies in AD have identified five additional genes (CD33, the MS4A6-MS4A4 cluster, ATP-binding cassette-A7 [ $A B C A 7], \mathrm{CD} 2$-associated protein $[C D 2 A P]$, and EPH receptor A1 [EPHA1]), the products of which are all postulated to be involved in immune system regulation (reviewed in ref. 67). How these risk loci affect the functions of innate immune cells inside and outside of the CNS and how they increase susceptibility to neurodegeneration are not yet fully understood. Nevertheless, taken together, these studies provide strong genetic support for the concept that microglial dysfunction can directly influence the onset and progression of a variety of neurodegenerative disorders, including ALS/FTD.

\section{Microglia in ALS: lessons from SOD1}

Transgenic mice overexpressing mutant human SOD1 (mutSOD1) are the most commonly used model to study disease pathogenesis in ALS. These mice develop a progressive motor neuron disease that resembles the clinical and pathological features of human ALS. Studies have shown that overexpressing mutSOD1 only in motor neurons is not sufficient to fully manifest disease, indicating that other cell types are also required for neurodegeneration (68-71). An elegant study using chimeric mice further supported the importance of non-neuronal cells in motor neuron death: motor neurons lacking mutSOD1 developed features of ALS pathology when surrounded by mutSOD1-expressing glia, whereas motor neurons expressing mutSOD1 but surrounded by WT glia appeared healthy (72). While astrocytes, oligodendrocytes, and other cells contribute to disease pathogenesis, several studies support that microglia play an important role in non-cell-autonomous motor neuron degeneration.

Evidence from cultured microglia supports that mutSOD1 can have a cell-autonomous effect on their function, rendering them capable of promoting motor neuron death. LPS-activated WT microglia are cytotoxic to cocultured primary motor neurons owing to production of ROS (73). LPS-stimulated microglial cells carrying mutSOD1 are at baseline more activated than WT microglia, produce more superoxide, $\mathrm{NO}$, and $\mathrm{TNF}-\alpha$, and are more injurious to primary cultured motor neurons $(74,75)$. Inhibition of $\mathrm{NF}-\kappa \mathrm{B}$ suppresses microglia-mediated neuroinflammatory toxicity when mutSOD1 microglial cells are cocultured with motor neurons (76). These results are further supported by data from transgenic mouse models overexpressing mutSOD1. In these animals, microglial activation in the spinal cord is evident prior to the onset of clinical weakness, increases as disease progresses, and is maintained through the late stages of disease $(77,78)$. Supporting that mutSOD1 can directly alter microglial function, removal of mutSOD1 transgene within Cd11 $b^{+}$cells increased the lifespan of SOD $1^{\mathrm{G} 37 \mathrm{R}}$ mice by approximately 100 days (79). Similarly, WT microglia extended survival in PU.1 knockout mice bred with SOD1 ${ }^{\mathrm{G} 93 \mathrm{~A}}$ mutants (80). Inhibition of IL-1 $\beta$, which is produced by activated microglia, also attenuated inflammatory pathology and enhanced survival in mutSOD1 animals (81), supporting that microglial-produced cytokines directly influence disease progression.

A key question that arises from these studies is how microglia become activated in the first place. Microglia are the resident 
A

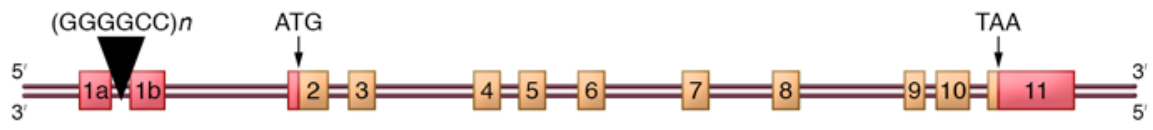

B

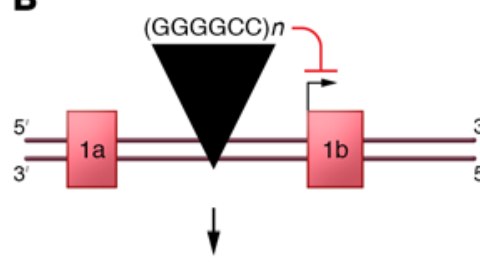

Loss of function/haploinsufficiency

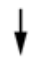

Decreased C9orf72 expression

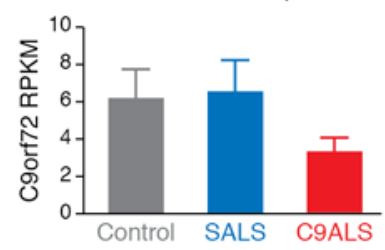

(Human frontal cortex)
RNA foci
C
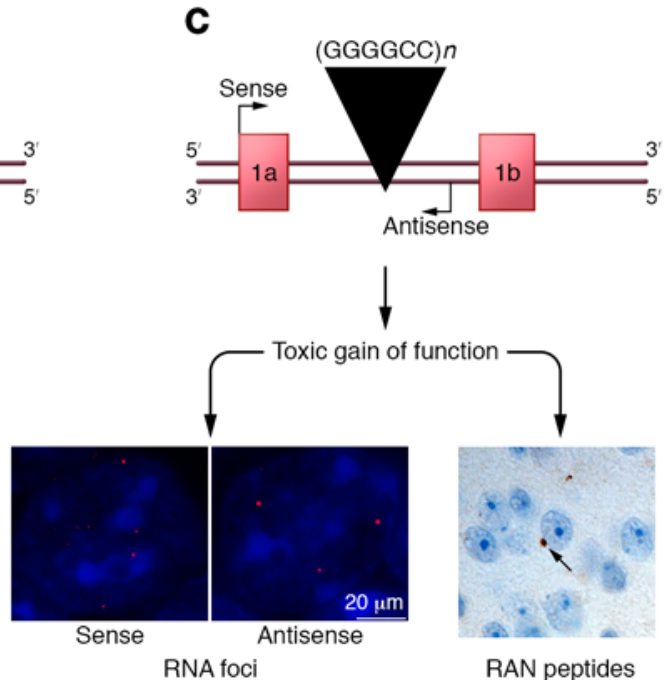

RAN peptides

Figure 1. Schematic of the C9orf72 gene. The location of the intronic hexanucleotide (GGGGCC) repeat expansion (black triangles) is shown between the noncoding exons $1 \mathrm{a}$ and $1 \mathrm{~b}$, upstream of the start codon. (A) Expansion of the hexanucleotide repeat from the normal range (about 2-30) to very large sizes (typically about $100-1,000$ ) is the most common genetic cause of ALS/FTD. Bars represent coding (yellow) and noncoding (red) regions, and the positions of the start codon (ATC) and stop codon (TAA) are indicated. (B) Large GGGGCC expansions cause downregulation of C9orf72 gene expression, supporting that loss of normal C9orf72 function may contribute to disease. SALS, sporadic ALS; C9ALS, C9-associated ALS. RPKM, reads per kilobase per million. (C) Both sense and antisense transcripts containing C9orf72 expansions form RNA foci in cell nuclei, which may have a toxic effect on nuclear protein function. Repeat-containing transcripts can also serve as templates for RAN translation to generate simple dipeptide repeat proteins (RAN peptides) that accumulate in the brains of carriers and may contribute to disease through a variety of toxic mechanisms. C9orf72 expression data are based on data from Prudencio et al. (112). Sense and antisense foci and RAN dipeptide pictures are reproduced with permission from Neuron (147).

surveillance cells of the brain and are highly sensitive to changes in their environment. They can respond to endogenous danger signals derived from molecules released by damaged cells, stressinduced proteins, and abnormal protein accumulation in their surroundings to initiate an immune response. In the context of ALS, one possibility is that accumulation of misfolded proteins (such as mutSOD1) in diseased motor neurons may activate microglia via release of different signaling molecules or the mutant protein itself. Studies have shown that mutant or oxidized SOD1 can activate microglia through CD14/TLR2/TLR4 and scavenger receptor-dependent pathways (82-84). Expression of mutSOD1 in microglia is also known to produce excessive extracellular superoxide (85), which can damage the nearby neurons. It is important to note, however, that once activated, microglia can manifest either protective or damaging effects depending on stage and progression of the disease. For example, microglia from presymptomatic mutSOD1 mice exhibit an antiinflammatory phenotype and attenuated innate immune response compared with microglia from symptomatic mice, which are proinflammatory and exhibit toxic properties $(86,87)$. Similar findings were demonstrated in vitro (88), suggesting that an adaptive shift in functional microglial phenotypes from neuroprotective to neurotoxic may accelerate disease progression in later stages.

\section{C9orf72 in ALS/FTD}

In 2011, the pathogenic expansion of a noncoding hexanucleotide repeat (GGGGCC) in C9orf72 was identified as the most common genetic cause of ALS and FTD, accounting for roughly $40 \%$ of familial ALS and $5 \%-10 \%$ of sporadic ALS cases $(11,14)$. Normal healthy individuals usually have 2-23 repeats, while affected individuals typically have repeat expansions in the hundreds or thousands. Given the major contribution of this repeat expansion mutation to both sporadic and familial ALS, there has been intense interest in deciphering the mechanisms by which GGGGCC repeat expansions cause neurodegeneration (89). Currently, three broad mechanisms are proposed to explain how these repeat expansions could cause disease (Figure 1). First, the presence of a large GGGGCC repeat expansion in the promoter region could cause a downregulation in C9orf72 expression, leading to a loss of C9orf72's normal cellular function. Many reports have observed decreased C9orf 72 transcript levels in tissues from mutation carriers $(11,90)$. Supporting the idea that haploinsufficiency could contribute to disease, complete loss of the Caenorhabditis elegans C9orf 72 ortholog resulted in motor neuron degeneration and age-dependent deficits in motility (91). These mutants were also hypersensitive to environmental stress-induced neurodegeneration. Similarly, depletion of C9orf 72 via antisense oligonucleotides in zebrafish resulted in axonal and motor defects, suggesting that loss of C9orf72 can directly impair motor neuron function (92). Second, RNA-mediated toxicity has been proposed due to the presence of sense (11) and antisense RNA foci (93) observed in cells from C9orf72 carrier brain and spinal cord samples. These RNA foci could sequester RNA-binding proteins, leading to splicing defects and alterations in RNA metabolism (94), but the exact identity of the proteins that bind to foci and are functionally altered remains elusive (95-97). Third, dipeptide repeat proteins that are produced by repeat-associated non-AUG (RAN) translation accumulate in the brain and spinal cord of C9orf 72 mutation carriers $(93,98,99)$ and have a variety of toxic properties that may drive neurodegeneration. Dipeptide repeat protein accumulations and/or RNA inclusions have been shown to impair nucleocytoplasmic transport in affected cells (100-102); however, ongoing studies are needed to determine the connection between nucleocytoplasmic transport defects and selective neuronal death in ALS/FTD. Potential gain-of-function mechanisms were recently reviewed elsewhere $(103,104)$. 
Loss of function

Allele 1
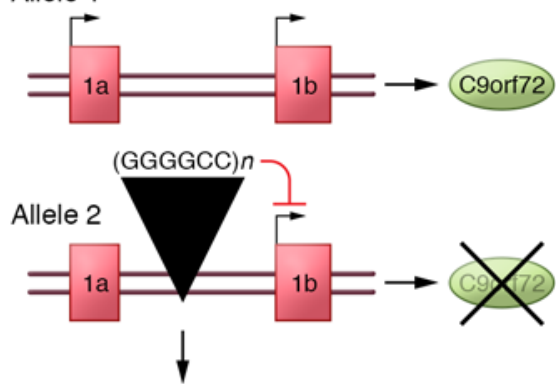

Microglia (and other cell types?)

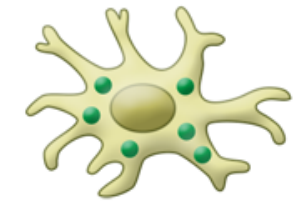

Endo-/lysosome dysfunction

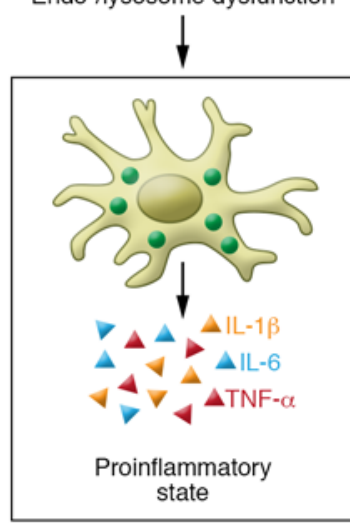

Gain of function

Allele 1

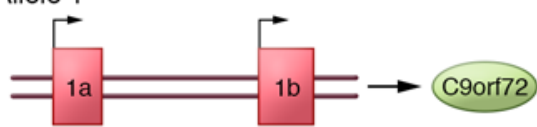

(GGGGCC)n

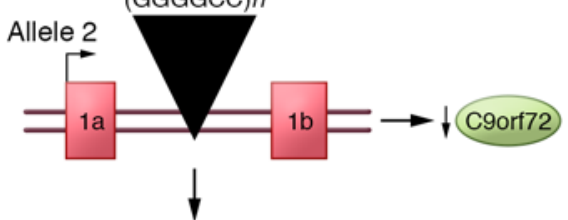

Neurons (and other cell types?)

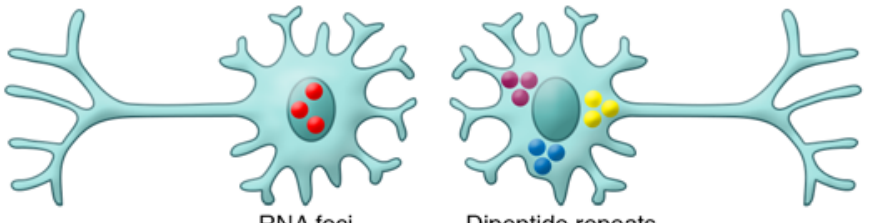

Dipeptide repeats
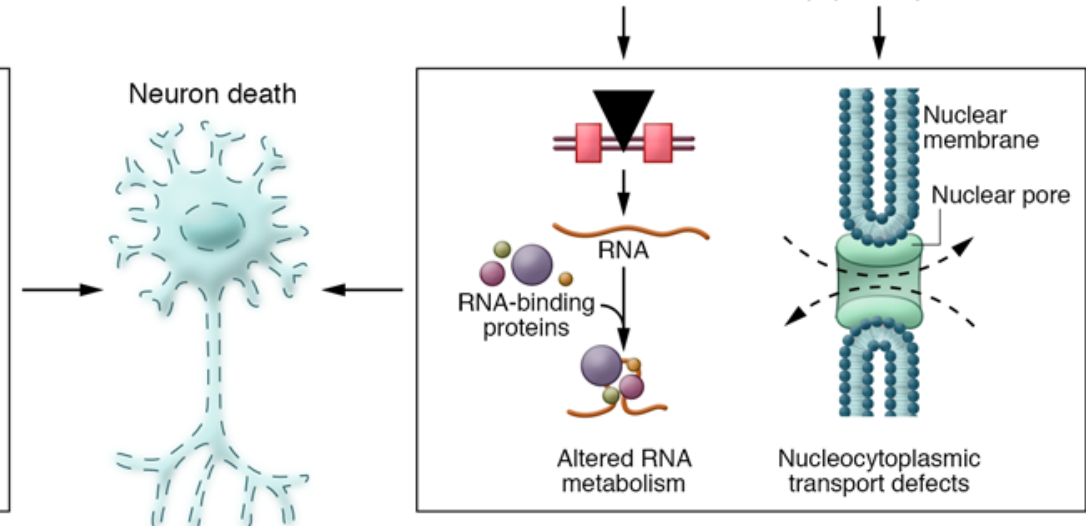

$\downarrow$

ALS/FTD

Figure 2. Combined model of loss and gain of function in C9orf72-related neurodegeneration. The repeat expansion leads to decreased C9orf72 expression in microglia (and potentially other cells), resulting in endosomal-lysosomal dysfunction, and promoting a proinflammatory state. By contrast, neurons transcribe the repeat-containing allele, leading to accumulation of both RNA foci and RAN dipeptides, which manifest a variety of toxic effects on neuronal function, including sequestration of RNA-binding proteins and altered RNA metabolism, and disruption of nucleocytoplasmic transport. The "dual effect" of the C9orf72 repeat expansion on different cell types (loss of function in microglia with a proinflammatory state and gain of function in neurons, leading to RNA and protein aggregate toxicity) may uniquely combine to promote neurodegeneration in ALS/FTD. Lysosomal accumulations seen in microglia are based on findings of O'Rourke et al. (106).

\section{C9orf72, myeloid cells, and immunity: an unexpected connection}

Several studies of germline knockout of C9orf72 in mice were published last year that provide insights into how haploinsufficiency of C9orf72 could contribute to neurodegeneration (105107). None of the mice in these studies showed neurodegeneration or motor system dysfunction consistent with ALS/FTD phenotypes, suggesting that C9orf72 function is not as critical in mammalian neurons as was observed in lower organisms. Instead, C9orf $72^{--}$mice developed progressive splenomegaly and lymphadenopathy, altered splenic myeloid cell populations, increased levels of circulating proinflammatory cytokines, and in some colonies a fulminant autoimmune disorder $(105,106,108)$. The effects seen upon C9orf 72 deletion are perhaps not surpris- ing since C9orf72 is highly expressed in myeloid cells, which are critically important for antigen presentation and development of autoimmunity, and loss of C9orf72 led to lysosomal accumulation and hyperactive immune responses in bone marrow macrophages (106, 109-111). The studies of C9orf72-null mice showed variability in the degree of autoantibody production and autoimmune-mediated tissue injury, indicating an environmental influence from different housing conditions that contributed to the development of autoimmunity. Interestingly, even heterozygous C9orf72 (C9orf $\left.72^{+/}\right)$animals showed a limited repertoire of autoantibodies with increased risk of early mortality (107), and bone marrow-derived macrophages from $C 9$ orf $72^{+-}$mice also showed altered cytokine responses to a variety of immune stimuli (106). Together these studies support (a) that complete loss of 
C9orf72 leads to a systemic proinflammatory state likely driven by myeloid cells in the spleen and lymph nodes, which can result in autoimmune tissue injury depending on the environment; and (b) that haploinsufficiency is sufficient to alter myeloid cell function and systemic immunity in mice. The fact that loss of just one copy of C9orf 72 can disrupt myeloid cell function has important implications for C9orf72-associated ALS/FTD, given that dominantly inherited C9orf72 expansions show only partial rather than complete loss of C9orf72 expression.

In addition to altered systemic immunity, C9orf72-deficient mice developed mild age-related neuroinflammation $(93,94)$. Similar to what was observed in C9orf $72^{-/-}$macrophages, C9orf $72^{-/-}$ microglia showed lysosomal accumulation and a proinflammatory phenotype with increased expression of IL-6 and IL-1 $\beta$ (106). Transcriptional profiling of spinal cords from $C 90 \mathrm{rf} 72^{-/-}$animals revealed age-related upregulation of inflammatory pathways, which were more similar to C9-associated FTD patient tissue than to sporadic FTD tissue $(106,112)$. This suggests that C9-associated FTD patients have enhanced microglial responses compared with sporadic FTD patients, which is consistent with a prior study showing enhanced microglial pathology in C9-associated FTD cases (113). Interestingly, an earlier experiment knocking down C9orf72 expression using intrathecal administration of antisense oligonucleotides in mice also showed upregulation of microglial activation genes, including Trem2, C1qa, and TYRO protein tyrosine kinase binding protein (Tyrobp), again without evidence of neurodegeneration (114). Taken together, these studies support the idea that decreased C9orf 72 expression in microglia can directly alter their function and result in age-related neuroinflammation; however, in isolation, this altered microglial function is not sufficient to cause neurodegeneration, at least in rodents.

The findings of altered myeloid cell function and autoimmunity in C9orf72-deficient mice raise an interesting question - do C9orf72 expansion carriers (or ALS/FTD patients in general) have altered immune responses or a tendency to autoimmunity? Interestingly, a variety of studies have investigated this question, and support the somewhat surprising notion that ALS/FTD and autoimmunity may have a shared etiology, as outlined below.

\section{Inflammation and autoimmunity in ALS/FTD}

The idea that autoimmunity is associated with ALS has been appreciated for decades. Seminal studies in the 1980s showed that ALS patients have a high frequency of monoclonal paraproteinemia (115) and a high incidence of autoimmune disorders (116). Numerous IgG antibodies and complement proteins are found in sera (117) from ALS patients, and antibody titer was positively associated with increased disease severity (118). Monoclonal Igs were detectable in $60 \%$ of sporadic ALS patients (119), and autoantibodies against ganglioside GM1 and GD1a (120), sulfoglucuronylparagloboside (121), neurofilament proteins (121), the TNF receptor family member FAS (CD95) (122), and voltage-gated $\mathrm{Ca}^{2+}$ channels $(123,124)$ have all been reported, indicating that ALS patients generate antibody responses to a variety of autoantigens.

More recent epidemiological and genetic studies continue to support the association between ALS and autoimmunity. A large epidemiological study found that there were significantly more cases than expected of ALS associated with a prior diagnosis of asthma, celiac disease, juvenile-onset diabetes, multiple sclerosis (MS), myasthenia gravis, systemic lupus erythematosus, and ulcerative colitis, supporting that autoimmune diseases and ALS might share common genetic or environmental risk factors (125). Other recent studies found an increased prevalence of autoimmune diseases in FTD with TDP-43 pathology (126), and specifically in C9orf 72 patients with ALS/FTD (127). Interestingly, in a small cohort of patients with the rare combination of MS and ALS, approximately $80 \%$ carried a hexanucleotide repeat expansion in C9orf72 (128), suggesting these patients may have hyperactive immune responses to myelin antigens. Likewise, the recent identification of loss-of-function mutations in TBK1, a well-characterized regulator of innate immunity, as a cause of ALS/FTD $(18,129$, 130) further reinforces the concept that immune dysregulation might be a characteristic feature of the disease.

Despite the strong case for a connection between ALS/FTD and autoimmunity, there is also convincing evidence against the idea that ALS is itself a classical autoimmune condition, given that administration of immunosuppressive drugs including corticosteroids, azathioprine, and cyclophosphamide (alone or in combination), as well as plasmapheresis or intravenous Igs (IVIGs), has failed to alter the progression of the disease (131-137). Several reasons have been posited to explain the failure of these drugs to slow disease progression in ALS patients. First, given that ALS is a heterogeneous disease, perhaps not all ALS patients have an inflammatory component. Second, since we do not know the dynamics and nature of the interaction between the immune system dysfunction and motor neuron death, we do not know whether administration of immunomodulatory therapies after disease onset may be too late, or even at a time when immune activation has a protective function. Finally, it is possible that instead of autoimmunity directly causing ALS/FTD, the two conditions arise from shared genetic or environmental risk factors and simply co-occur in the same individuals and families.

Owing to the dual effects of microglia in the CNS, being either protective or detrimental depending on expression of regulatory signals released in the local environment at different disease stages, targeting microglia therapeutically presents significant challenges. Additional longitudinal studies with comprehensive phenotyping of the immune system and a better understanding of how emerging genetic risk factors including C9orf72 and TBK1 influence immune cell function will be helpful to rationally design and test immunotherapies in ALS/FTD.

\section{C9orf72 loss of function and inflammation: looking forward}

Despite the explosion in research on the role of C9orf72 expansion in neurodegeneration, we have much to learn about the potential loss- and gain-of-function effects of C9orf 72 on both neuroinflammation and neurodegeneration. Studies deciphering the molecular functions of C9orf72 are helping define the effects of loss in different cell types, including neurons and microglia. Bioinformatic analysis has shown that $\mathrm{C} 9$ orf72 contains a differentially expressed in normal and neoplastic cells (DENN) domain (138) and is hypothesized to play a role in intracellular trafficking and autophagy and lysosomal functions. Moreover, upon C 9 orf 72 depletion, endocytosis was found to be impaired and autophago- 
some formation to be dysregulated (139), suggesting that C9orf72 plays a critical role in autophagy and endosomal-lysosomal pathways. C9orf72 was also shown to interact with the TBK1 targets Smith-Magenis syndrome chromosome region, candidate 8 (SMCR8), and WD repeat domain 41 (WDR41) (140-142), suggesting that C9orf72 and TBK1 may act within the same pathway. As discussed earlier, mutations in a number of genes known to be involved in autophagy and lysosomal pathways are linked to ALS/ FTD, including TBK1, OPTN, VCP, PGRN, UBQLN2, charged multivesicular body protein 2B (CHMP2B), and SQSTM1 (8-10, $129,143,144)$. The ubiquitous presence of ubiquitin- and p62positive inclusions in ALS/FTD cases further supports the idea that an impaired autophagosome-lysosome system plays a role in disease. Given that these genes are expressed in both microglia and neurons, it is possible that mutations in this cluster of ALS/ FTD genes share a common ability to promote aberrant protein aggregation in neurons (toxic gain of function) and abnormal inflammatory responses in microglia (loss of C9orf72 function), which uniquely combine to result in neurodegeneration (Figure 2). Likewise, alterations in this gene cluster may be partly responsible for the shared tendency toward both autoimmunity and neurodegeneration in ALS/FTD. Continued experimental work is needed to address some key questions moving forward. First, which cell types exhibit loss- and gain-of-function manifestations of C9orf72 expansion in human patient tissues, and do some cells manifest both gain and loss of function? Studies on brain tissue showing reduced C9orf72 expression have primarily looked at the RNA level, and were not able to determine which cell types contribute to the overall decrease in expression. Moving forward it will be helpful to incorporate better antibodies to examine protein levels, and to examine expression and epigenetic modification at the cellular rather than the tissue level. Second, can altered immune responses be measured in cells from C9orf 72 expansion carriers, and are loss-of-function mutations in C9orf 72 enriched in any autoimmune conditions? Such data would help us to understand whether the decrease in C9orf 72 expression (around 50\%) seen in patients has similar immunological consequences to those observed in the rodent models. Third, can combined lossand gain-of-function alterations in C9orf 72 be demonstrated in cell- or animal-based experimental models?

Answering these questions will have immediate implications for therapeutics, given that one of the primary strategies being developed to treat C9orf72-associated ALS/FTD is knockdown of the toxic $C 9$ orf 72 species using antisense oligonucleotide technology $(114,145,146)$. Therefore, a deeper understanding of the effect of loss of C9orf72 function on immune cells will be important both to interpret the results of knockdown studies in humans and to properly monitor patients for potential adverse effects.

\section{Acknowledgments}

This work was supported by NIH grants NS097545 and NS069669 (to RHB), the Robert and Louise Schwab family, Target ALS, the Cedars-Sinai ALS Research Fund, and the Cedars-Sinai Board of Governors Regenerative Medicine Institute.

Address correspondence to: Robert H. Baloh, Cedars-Sinai Medical Center, 8700 Beverly Boulevard, Los Angeles, California 90048, USA. Phone: 310.423.1525; Email: robert.baloh@csmc.edu.
1. Haverkamp LJ, Appel V, Appel SH. Natural history of amyotrophic lateral sclerosis in a database population. Validation of a scoring system and a model for survival prediction. Brain. 1995;118(pt 3):707-719.

2. Boillée S, Vande Velde C, Cleveland DW. ALS: a disease of motor neurons and their nonneuronal neighbors. Neuron. 2006;52(1):39-59.

3. Rosen DR. Mutations in $\mathrm{Cu} / \mathrm{Zn}$ superoxide dismutase gene are associated with familial amyotrophic lateral sclerosis. Nature. 1993;364(6435):362.

4. Sreedharan J, et al. TDP-43 mutations in familial and sporadic amyotrophic lateral sclerosis. Science. 2008;319(5870):1668-1672.

5 . Vance C, et al. Mutations in FUS, an RNA processing protein, cause familial amyotrophic lateral sclerosis type 6. Science. 2009;323(5918):1208-1211.

6. Kwiatkowski TJ, et al. Mutations in the FUS/TLS gene on chromosome 16 cause familial amyotrophic lateral sclerosis. Science. 2009;323(5918):1205-1208.

7. Deng HX, et al. Differential involvement of optineurin in amyotrophic lateral sclerosis with or without SOD1 mutations. Arch Neurol. 2011;68(8):1057-1061.

8. Maruyama $\mathrm{H}$, et al. Mutations of optineurin in amyotrophic lateral sclerosis. Nature. 2010;465(7295):223-226.

9. Johnson JO, et al. Exome sequencing reveals VCP mutations as a cause of familial ALS. Neuron.
2010;68(5):857-864.

10. Fecto F, et al. SQSTM1 mutations in familial and sporadic amyotrophic lateral sclerosis. Arch Neurol.2011;68(11):1440-1446.

11. DeJesus-Hernandez M, et al. Expanded GGGGCC hexanucleotide repeat in noncoding region of C9ORF72 causes chromosome 9p-linked FTD and ALS. Neuron. 2011;72(2):245-256.

12. Kim HJ, et al. Mutations in prion-like domains in hnRNPA2B1 and hnRNPA1 cause multisystem proteinopathy and ALS. Nature. 2013;495(7442):467-473.

13. Liu Q, et al. Whole-exome sequencing identifies a missense mutation in hnRNPA1 in a family with flail arm ALS. Neurology. 2016;87(17):1763-1769.

14. Renton AE, et al. A hexanucleotide repeat expansion in C9ORF72 is the cause of chromosome 9p21-linked ALS-FTD. Neuron. 2011;72(2):257-268

15. Kabashi E, et al. TARDBP mutations in individuals with sporadic and familial amyotrophic lateral sclerosis. Nat Genet. 2008;40(5):572-574.

16. Teyssou E, et al. Mutations in SQSTM1 encoding p62 in amyotrophic lateral sclerosis: genetics and neuropathology. Acta Neuropathol. 2013;125(4):511-522.

17. $\mathrm{Wu} \mathrm{CH}$, et al. Mutations in the profilin 1 gene cause familial amyotrophic lateral sclerosis. Nature. 2012;488(7412):499-503.

18. Freischmidt A, et al. Haploinsufficiency of TBK1 causes familial ALS and fronto-temporal demen- tia. Nat Neurosci. 2015;18(5):631-636.

19. Cooper-Knock J, Kirby J, Ferraiuolo L, Heath PR, Rattray M, Shaw PJ. Gene expression profiling in human neurodegenerative disease. Nat Rev Neurol. 2012;8(9):518-530.

20. Cooper-Knock J, et al. Clinico-pathological features in amyotrophic lateral sclerosis with expansions in C9ORF72. Brain. 2012;135(pt 3):751-764.

21. Cady J, et al. Amyotrophic lateral sclerosis onset is influenced by the burden of rare variants in known amyotrophic lateral sclerosis genes. Ann Neurol. 2015;77(1):100-113.

22. Elden AC, et al. Ataxin-2 intermediate-length polyglutamine expansions are associated with increased risk for ALS. Nature. 2010;466(7310):1069-1075.

23. Van Hoecke A, et al. EPHA4 is a disease modifier of amyotrophic lateral sclerosis in animal models and in humans. Nat Med. 2012;18(9):1418-1422.

24. Robberecht W, Philips T. The changing scene of amyotrophic lateral sclerosis. Nat Rev Neurosci. 2013;14(4):248-264.

25. Ferraiuolo L, Kirby J, Grierson AJ, Sendtner M, Shaw PJ. Molecular pathways of motor neuron injury in amyotrophic lateral sclerosis. Nat Rev Neurol. 2011;7(11):616-630.

26. Corcia P, et al. Molecular imaging of microglial activation in amyotrophic lateral sclerosis. PLOS ONE. 2012;7(12):e52941.

27. Turner MR, et al. Evidence of widespread cerebral microglial activation in amyotrophic 
lateral sclerosis: an $\left[{ }^{11} \mathrm{C}\right](\mathrm{R})-\mathrm{PK} 11195$ positron emission tomography study. Neurobiol Dis. 2004;15(3):601-609.

28. Engelhardt JI, Appel SH. IgG reactivity in the spinal cord and motor cortex in amyotrophic lateral sclerosis. Arch Neurol. 1990;47(11):1210-1216.

29. Engelhardt JI, Tajti J, Appel SH. Lymphocytic infiltrates in the spinal cord in amyotrophic lateral sclerosis. Arch Neurol. 1993;50(1):30-36.

30. Henkel JS, et al. Presence of dendritic cells, MCP-1, and activated microglia/macrophages in amyotrophic lateral sclerosis spinal cord tissue. Ann Neurol. 2004;55(2):221-235.

31. Cruts M, et al. Null mutations in progranulin cause ubiquitin-positive frontotemporal dementia linked to chromosome 17q21. Nature. 2006;442(7105):920-924.

32. Pickering-Brown SM, et al. Mutations in progranulin explain atypical phenotypes with variants in MAPT. Brain. 2006;129(pt 11):3124-3126.

33. Gass J, et al. Mutations in progranulin are a major cause of ubiquitin-positive frontotemporal lobar degeneration. Hum Mol Genet. 2006;15(20):2988-3001.

34. Matcovitch-Natan O, et al. Microglia development follows a stepwise program to regulate brain homeostasis. Science. 2016;353(6301):aad8670.

35. Town T, Nikolic V, Tan J. The microglial "activation" continuum: from innate to adaptive responses. J Neuroinflammation. 2005;2:24.

36. Schafer DP, Stevens B. Microglia function in central nervous system development and plasticity. Cold Spring Harb Perspect Biol. 2015;7(10):a020545.

37. Ginhoux F, Lim S, Hoeffel G, Low D, Huber T. Origin and differentiation of microglia. Front Cell Neurosci. 2013;7:45.

38. Bennett ML, et al. New tools for studying microglia in the mouse and human CNS. Proc Natl Acad Sci U S A. 2016;113(12):E1738-E1746.

39. London A, Cohen M, Schwartz M. Microglia and monocyte-derived macrophages: functionally distinct populations that act in concert in CNS plasticity and repair. Front Cell Neurosci. 2013;7:34.

40. Popovich PG, Hickey WF. Bone marrow chimeric rats reveal the unique distribution of resident and recruited macrophages in the contused rat spinal cord. J Neuropathol Exp Neurol. 2001;60(7):676-685.

41. Ajami B, Bennett JL, Krieger C, McNagny KM, Rossi FM. Infiltrating monocytes trigger EAE progression, but do not contribute to the resident microglia pool. Nat Neurosci. 2011;14(9):1142-1149.

42. Hanisch UK, Kettenmann H. Microglia: active sensor and versatile effector cells in the normal and pathologic brain. Nat Neurosci. 2007;10(11):1387-1394.

43. Neumann H, Kotter MR, Franklin RJ. Debris clearance by microglia: an essential link between degeneration and regeneration. Brain. 2009;132(Pt 2):288-295.

44. Malik M, et al. Genetics ignite focus on microglial inflammation in Alzheimer's disease. Mol Neurodegener. 2015;10:52.

45. Holmans P, et al. A pathway-based analysis pro- vides additional support for an immune-related genetic susceptibility to Parkinson's disease. Hum Mol Genet. 2013;22(5):1039-1049.

46. Dzamko N, Geczy CL, Halliday GM. Inflammation is genetically implicated in Parkinson's disease. Neuroscience. 2015;302:89-102.

47. Cady J, et al. TREM2 variant p.R47H as a risk factor for sporadic amyotrophic lateral sclerosis. JAMA Neurol. 2014;71(4):449-453.

48. Colonna M. TREMs in the immune system and beyond. Nat Rev Immunol. 2003;3(6):445-453.

49. Ulrich JD, Holtzman DM. TREM2 function in Alzheimer's disease and neurodegeneration. ACS Chem Neurosci. 2016;7(4):420-427.

50. Thrash JC, Torbett BE, Carson MJ. Developmental regulation of TREM2 and DAP12 expression in the murine CNS: implications for Nasu-Hakola disease. Neurochem Res. 2009;34(1):38-45.

51. Wang Y, et al. TREM2 lipid sensing sustains the microglial response in an Alzheimer's disease model. Cell. 2015;160(6):1061-1071.

52. Kleinberger G, et al. TREM2 mutations implicated in neurodegeneration impair cell surface transport and phagocytosis. Sci Transl Med. 2014;6(243):243ra86.

53. Petkau TL, Leavitt BR. Progranulin in neurodegenerative disease. Trends Neurosci. 2014;37(7):388-398.

54. Pickford F, et al. Progranulin is a chemoattractant for microglia and stimulates their endocytic activity. Am J Pathol. 2011;178(1):284-295.

55. Minami SS, et al. Progranulin protects against amyloid $\beta$ deposition and toxicity in Alzheimer's disease mouse models. Nat Med. 2014;20(10):1157-1164.

56. Lui H, et al. Progranulin deficiency promotes circuit-specific synaptic pruning by microglia via complement activation. Cell. 2016;165(4):921-935.

57. Lee H, James WS, Cowley SA. LRRK2 in peripheral and central nervous system innate immunity: its link to Parkinson's disease. Biochem Soc Trans. 2017;45(1):131-139.

58. Liu CC, Liu CC, Kanekiyo T, Xu H, Bu G. Apolipoprotein $\mathrm{E}$ and Alzheimer disease: risk, mechanisms and therapy. Nat Rev Neurol. 2013;9(2):106-118.

59. Schafer DP, et al. Microglia sculpt postnatal neural circuits in an activity and complementdependent manner. Neuron. 2012;74(4):691-705

60. Hong S, Dissing-Olesen L, Stevens B. New insights on the role of microglia in synaptic pruning in health and disease. Curr Opin Neurobiol. 2016;36:128-134.

61. Benoit ME, Hernandez MX, Dinh ML, Benavente F, Vasquez O, Tenner AJ. C1q-induced LRP1B and GPR6 proteins expressed early in Alzheimer disease mouse models, are essential for the C1q-mediated protection against amyloid- $\beta$ neurotoxicity. J Biol Chem. 2013;288(1):654-665.

62. Alexander JJ, Anderson AJ, Barnum SR, Stevens B, Tenner AJ. The complement cascade: Yin-Yang in neuroinflammation - neuroprotection and -degeneration. JNeurochem. 2008;107(5):1169-1187.

63. Hong S, et al. Complement and microglia mediate early synapse loss in Alzheimer mouse models. Science. 2016;352(6286):712-716.
64. Liddelow SA, et al. Neurotoxic reactive astrocytes are induced by activated microglia. Nature. 2017;541(7638):481-487.

65. Lambert JC, et al. Genome-wide haplotype association study identifies the FRMD4A gene as a risk locus for Alzheimer's disease. Mol Psychiatry. 2013;18(4):461-470.

66. DeMattos RB, et al. ApoE and clusterin cooperatively suppress Abeta levels and deposition: evidence that ApoE regulates extracellular Abeta metabolism in vivo. Neuron. 2004;41(2):193-202.

67. Karch CM, Goate AM. Alzheimer's disease risk genes and mechanisms of disease pathogenesis. Biol Psychiatry. 2015;77(1):43-51.

68. Pramatarova A, Laganière J, Roussel J, Brisebois K, Rouleau GA. Neuron-specific expression of mutant superoxide dismutase 1 in transgenic mice does not lead to motor impairment. J Neurosci. 2001;21(10):3369-3374.

69. Lino MM, Schneider C, Caroni P. Accumulation of SOD1 mutants in postnatal motoneurons does not cause motoneuron pathology or motoneuron disease. J Neurosci. 2002;22(12):4825-4832.

70. Yamanaka K, et al. Mutant SOD1 in cell types other than motor neurons and oligodendrocytes accelerates onset of disease in ALS mice. Proc Natl Acad Sci U S A. 2008;105(21):7594-7599.

71. Jaarsma D, Teuling E, Haasdijk ED, De Zeeuw CI, Hoogenraad CC. Neuron-specific expression of mutant superoxide dismutase is sufficient to induce amyotrophic lateral sclerosis in transgenic mice. J Neurosci. 2008;28(9):2075-2088.

72. Clement AM, et al. Wild-type nonneuronal cells extend survival of SOD1 mutant motor neurons in ALS mice. Science. 2003;302(5642):113-117.

73. Zhao W, et al. Activated microglia initiate motor neuron injury by a nitric oxide and glutamatemediated mechanism. J Neuropathol Exp Neurol. 2004;63(9):964-977.

74. Xiao Q, et al. Mutant SOD1(G93A) microglia are more neurotoxic relative to wild-type microglia. J Neurochem. 2007;102(6):2008-2019.

75. Weydt P, Yuen EC, Ransom BR, Möller T. Increased cytotoxic potential of microglia from ALS-transgenic mice. Glia. 2004;48(2):179-182.

76. Frakes AE, et al. Microglia induce motor neuron death via the classical NF- $\mathrm{BB}$ pathway in amyotrophic lateral sclerosis. Neuron. 2014;81(5):1009-1023.

77. Alexianu ME, Kozovska M, Appel SH. Immune reactivity in a mouse model of familial ALS correlates with disease progression. Neurology. 2001;57(7):1282-1289.

78. Hall ED, Oostveen JA, Gurney ME. Relationship of microglial and astrocytic activation to disease onset and progression in a transgenic model of familial ALS. Glia. 1998;23(3):249-256.

79. Boillée $S$, et al. Onset and progression in inherited ALS determined by motor neurons and microglia. Science. 2006;312(5778):1389-1392.

80. Beers DR, et al. Wild-type microglia extend survival in PU.1 knockout mice with familial amyotrophic lateral sclerosis. Proc Natl Acad Sci US A. 2006;103(43):16021-16026.

81. Meissner F, Molawi K, Zychlinsky A. Mutant superoxide dismutase 1-induced IL-1beta accelerates ALS pathogenesis. Proc Natl Acad Sci US A. 2010;107(29):13046-13050. 
82. Ezzi SA, Urushitani M, Julien JP. Wild-type superoxide dismutase acquires binding and toxic properties of ALS-linked mutant forms through oxidation. J Neurochem. 2007;102(1):170-178.

83. Roberts K, Zeineddine R, Corcoran L, Li W, Campbell IL, Yerbury JJ. Extracellular aggregated $\mathrm{Cu} / \mathrm{Zn}$ superoxide dismutase activates microglia to give a cytotoxic phenotype. Glia. 2013;61(3):409-419.

84. Zhao W, et al. Extracellular mutant SOD1 induces microglial-mediated motoneuron injury. Glia. 2010;58(2):231-243.

85. Harraz MM, et al. SOD1 mutations disrupt redox-sensitive Rac regulation of NADPH oxidase in a familial ALS model. J Clin Invest. 2008;118(2):659-670.

86. Gravel M, et al. IL-10 controls early microglial phenotypes and disease onset in ALS caused by misfolded superoxide dismutase 1. J Neurosci. 2016;36(3):1031-1048.

87. Henkel JS, Beers DR, Zhao W, Appel SH. Microglia in ALS: the good, the bad, and the resting. J Neuroimmune Pharmacol. 2009;4(4):389-398.

88. Liao B, Zhao W, Beers DR, Henkel JS, Appel SH. Transformation from a neuroprotective to a neurotoxic microglial phenotype in a mouse model of ALS. Exp Neurol. 2012;237(1):147-152.

89. Ling SC, Polymenidou M, Cleveland DW. Converging mechanisms in ALS and FTD: disrupted RNA and protein homeostasis. Neuron. 2013;79(3):416-438.

90. Gijselinck I, et al. A C9orf72 promoter repeat expansion in a Flanders-Belgian cohort with disorders of the frontotemporal lobar degeneration-amyotrophic lateral sclerosis spectrum: a gene identification study. Lancet Neurol. 2012;11(1):54-65.

91. Therrien M, Rouleau GA, Dion PA, Parker JA. Deletion of C9ORF72 results in motor neuron degeneration and stress sensitivity in C. elegans. PLoS One. 2013;8(12):e83450.

92. Ciura S, et al. Loss of function of C9orf72 causes motor deficits in a zebrafish model of amyotrophic lateral sclerosis. Ann Neurol. 2013;74(2):180-187.

93. Gendron TF, et al. Antisense transcripts of the expanded C9ORF72 hexanucleotide repeat form nuclear RNA foci and undergo repeat-associated non-ATG translation in c9FTD/ALS. Acta Neuropathol. 2013;126(6):829-844.

94. La Spada AR, Taylor JP. Repeat expansion disease: progress and puzzles in disease pathogenesis. Nat Rev Genet. 2010;11(4):247-258.

95. Haeusler AR, et al. C9orf72 nucleotide repeat structures initiate molecular cascades of disease. Nature. 2014;507(7491):195-200.

96. Lee YB, et al. Hexanucleotide repeats in ALS/ FTD form length-dependent RNA foci, sequester RNA binding proteins, and are neurotoxic. Cell Rep. 2013;5(5):1178-1186.

97. Xu Z, et al. Expanded GGGGCC repeat RNA associated with amyotrophic lateral sclerosis and frontotemporal dementia causes neurodegeneration. Proc Natl Acad Sci U S A. 2013;110(19):7778-7783.

98. Mackenzie IR, et al. Dipeptide repeat protein pathology in C9ORF72 mutation cases: clinicopathological correlations. Acta Neuropathol. 2013;126(6):859-879.
99. Ash PE, et al. Unconventional translation of C9ORF72 GGGGCC expansion generates insoluble polypeptides specific to c9FTD/ALS. Neuron. 2013;77(4):639-646.

100.Zhang $\mathrm{K}$, et al. The C9orf72 repeat expansion disrupts nucleocytoplasmic transport. Nature. 2015;525(7567):56-61.

101.Freibaum BD, et al. GGGGCC repeat expansion in C9orf72 compromises nucleocytoplasmic transport. Nature. 2015;525(7567):129-133.

102. Jovičić A, et al. Modifiers of C9orf72 dipeptide repeat toxicity connect nucleocytoplasmic transport defects to FTD/ALS. Nat Neurosci. 2015;18(9):1226-1229.

103. Taylor JP, Brown RH, Cleveland DW. Decoding ALS: from genes to mechanism. Nature. 2016;539(7628):197-206.

104. Gitler AD, Tsuiji H. There has been an awakening: Emerging mechanisms of C9orf72 mutations in FTD/ALS. Brain Res. 2016;1647:19-29.

105. Atanasio A, et al. C9orf72 ablation causes immune dysregulation characterized by leukocyte expansion, autoantibody production, and glomerulonephropathy in mice. Sci Rep. 2016;6:23204.

106.O'Rourke JG, et al. C9orf72 is required for proper macrophage and microglial function in mice. Science. 2016;351(6279):1324-1329.

107. Burberry A, et al. Loss-of-function mutations in the C9ORF72 mouse ortholog cause fatal autoimmune disease. Sci Transl Med. 2016;8(347):347ra93.

108. Sudria-Lopez E, et al. Full ablation of C9orf72 in mice causes immune system-related pathology and neoplastic events but no motor neuron defects. Acta Neuropathol. 2016;132(1):145-147.

109. Rizzu P, et al. C9orf72 is differentially expressed in the central nervous system and myeloid cells and consistently reduced in C9orf72, MAPT and GRN mutation carriers. Acta Neuropathol Commun. 2016;4(1):37.

110. Heng TS, Painter MW, Immunological Genome Project Consortium. The Immunological Genome Project: networks of gene expression in immune cells. Nat Immunol. 2008;9(10):1091-1094.

111. Nataf S, Pays L. Gene co-expression analysis unravels a link between C9orf72 and RNA metabolism in myeloid cells. Acta Neuropathol Commun. 2015;3:64.

112. Prudencio $M$, et al. Distinct brain transcriptome profiles in C9orf72-associated and sporadic ALS. Nat Neurosci. 2015;18(8):1175-1182.

113. Brettschneider J, et al. Microglial activation correlates with disease progression and upper motor neuron clinical symptoms in amyotrophic lateral sclerosis. PLoS One. 2012;7(6):e39216.

114. Lagier-Tourenne $\mathrm{C}$, et al. Targeted degradation of sense and antisense C9orf72 RNA foci as therapy for ALS and frontotemporal degeneration. Proc Natl Acad Sci U S A. 2013;110(47):E4530-E4539.

115. Shy ME, et al. Motor neuron disease and plasma cell dyscrasia. Neurology. 1986;36(11):1429-1436.

116. Appel SH, Stockton-Appel V, Stewart SS, Kerman RH. Amyotrophic lateral sclerosis. Associated clinical disorders and immunological evaluations. Arch Neurol. 1986;43(3):234-238.

117. Donnenfeld H, Kascsak RJ, Bartfeld H. Deposits of IgG and C3 in the spinal cord and motor cortex of ALS patients. J Neuroimmunol. 1984;6(1):51-57. 118. May C, et al. Highly immunoreactive IgG antibodies directed against a set of twenty human proteins in the sera of patients with amyotrophic lateral sclerosis identified by protein array. PLOS ONE. 2014;9(2):e89596.

119. Duarte F, Binet S, Lacomblez L, Bouche P, Preud'homme JL, Meininger V. Quantitative analysis of monoclonal immunoglobulins in serum of patients with amyotrophic lateral sclerosis. J Neurol Sci. 1991;104(1):88-91.

120. Pestronk A, Adams RN, Cornblath D, Kuncl RW, Drachman DB, Clawson L. Patterns of serum IgM antibodies to GM1 and GD1a gangliosides in amyotrophic lateral sclerosis. Ann Neurol. 1989;25(1):98-102.

121. Ben Younes-Chennoufi A, et al. Anti-sulfoglucuronyl paragloboside IgM antibodies in amyotrophic lateral sclerosis. J Neuroimmunol. 1995;57(1-2):111-115.

122. Yi FH, et al. In vitro induction of neuronal apoptosis by anti-Fas antibody-containing sera from amyotrophic lateral sclerosis patients. J Neuroimmunol. 2000;109(2):211-220.

123. Appel SH, et al. Increased intracellular calcium triggered by immune mechanisms in amyotrophic lateral sclerosis. Clin Neurosci. 1995;3(6):368-374.

124.Uchitel OD, Appel SH, Crawford F, Sczcupak L. Immunoglobulins from amyotrophic lateral sclerosis patients enhance spontaneous transmitter release from motor-nerve terminals. Proc Natl Acad Sci U S A. 1988;85(19):7371-7374.

125. Turner MR, Goldacre R, Ramagopalan S, Talbot K, Goldacre MJ. Autoimmune disease preceding amyotrophic lateral sclerosis: an epidemiologic study. Neurology. 2013;81(14):1222-1225.

126. Miller ZA, et al. TDP-43 frontotemporal lobar degeneration and autoimmune disease. J Neurol Neurosurg Psychiatr. 2013;84(9):956-962.

127. Miller ZA, et al. Increased prevalence of autoimmune disease within $\mathrm{C} 9$ and FTD/MND cohorts: Completing the picture. Neurol Neuroimmunol Neuroinflamm. 2016;3(6):e301.

128. Ismail A, et al. Concurrence of multiple sclerosis and amyotrophic lateral sclerosis in patients with hexanucleotide repeat expansions of C9ORF72. J Neurol Neurosurg Psychiatr. 2013;84(1):79-87.

129. Cirulli ET, et al. Exome sequencing in amyotrophic lateral sclerosis identifies risk genes and pathways. Science. 2015;347(6229):1436-1441.

130. Pottier C, et al. Whole-genome sequencing reveals important role for TBK1 and OPTN mutations in frontotemporal lobar degeneration without motor neuron disease. Acta Neuropathol. 2015;130(1):77-92.

131. Pagani MR, Gonzalez LE, Uchitel OD. Autoimmunity in amyotrophic lateral sclerosis: past and present. Neurol Res Int. 2011;2011:497080.

132. Baumann J. Results of treatment of certain diseases of the central nervous system with ACTH and corticosteroids. Acta Neurol Scand Suppl. 1965;1(pt 2):453-461.

133. Monstad I, Dale I, Petlund CF, Sjaastad O. Plasma exchange in motor neuron disease. A controlled study. J Neurol. 1979;221(1):59-66.

134. Olarte MR, Schoenfeldt RS, McKiernan G, Rowland LP. Plasmapheresis in amyotrophic lateral 
sclerosis. Ann Neurol. 1980;8(6):644-645.

135. Brown RH, Hauser SL, Harrington H, Weiner HL. Failure of immunosuppression with a 10- to 14-day course of high-dose intravenous cyclophosphamide to alter the progression of amyotrophic lateral sclerosis. Arch Neurol. 1986;43(4):383-384.

136. Appel SH, et al. A double-blind study of the effectiveness of cyclosporine in amyotrophic lateral sclerosis. Arch Neurol. 1988;45(4):381-386.

137. Drachman DB, et al. Trial of immunosuppression in amyotrophic lateral sclerosis using total lymphoid irradiation. Ann Neurol. 1994;35(2):142-150.

138. Levine TP, Daniels RD, Gatta AT, Wong LH, Hayes MJ. The product of C9orf72, a gene strongly implicated in neurodegeneration, is structurally related to DENN Rab-GEFs. Bioin- formatics. 2013;29(4):499-503.

139. Farg MA, et al. C9ORF72, implicated in amytrophic lateral sclerosis and frontotemporal dementia, regulates endosomal trafficking. Hum Mol Genet. 2014;23(13):3579-3595.

140. Sellier C, et al. Loss of C9ORF72 impairs autophagy and synergizes with polyQ Ataxin-2 to induce motor neuron dysfunction and cell death. ЕМВО J. 2016;35(12):1276-1297.

141. Sullivan PM, et al. The ALS/FTLD associated protein C9orf72 associates with SMCR8 and WDR41 to regulate the autophagylysosome pathway. Acta Neuropathol Commun. 2016;4(1):51.

142. Tang BL. C9orf72's interaction with Rab GTPases-modulation of membrane traffic and autophagy. Front Cell Neurosci. 2016;10:228.
143.Skibinski G, et al. Mutations in the endosomal ESCRTIII-complex subunit CHMP2B in frontotemporal dementia. Nat Genet. 2005;37(8):806-808.

144. Deng HX, et al. Mutations in UBQLN2 cause dominant X-linked juvenile and adult-onset ALS and ALS/dementia. Nature. 2011;477(7363):211-215.

145. Donnelly CJ, et al. RNA toxicity from the ALS/ FTD C9ORF72 expansion is mitigated by antisense intervention. Neuron. 2013;80(2):415-428.

146.Sareen D, et al. Targeting RNA foci in iPSCderived motor neurons from ALS patients with a C9ORF72 repeat expansion. Sci Transl Med. 2013;5(208):208ra149.

147. O'Rourke JG, et al. C9orf72 BAC transgenic mice display typical pathologic features of ALS/FTD. Neuron. 2015;88(5):892-901. 